\title{
Renal protective effect of the aortic balloon occlusion technique in total arch replacement with frozen elephant trunk
}

\author{
Bowen Zhang", Yanxiang Liu", Hongwei Guo, Yunfeng Li, Yi Shi, Shenghua Liang, Hong Liu, \\ Xiaogang Sun \\ Department of Cardiovascular Surgery, State Key Laboratory of Cardiovascular Disease, Fuwai Hospital, National Center for Cardiovascular \\ Diseases, Chinese Academy of Medical Sciences and Peking Union Medical College, Beijing 100037, China \\ \#These authors contributed to the work equally and should be regarded as co-first authors. \\ Correspondence to: Xiaogang Sun. Department of Cardiovascular Surgery, State Key Laboratory of Cardiovascular Disease, Fuwai Hospital, National \\ Center for Cardiovascular Diseases, Chinese Academy of Medical Sciences and Peking Union Medical College, No. 167 North Lishi Road, Xicheng \\ District, Beijing 100037, China. Email: xiaogangsun1@163.com.
}

Background: Organ dysfunction caused by hypothermic circulatory arrest continues to concern surgeons. The aortic balloon occlusion (ABO) technique can significantly shorten the circulatory arrest time in total arch replacement with frozen elephant trunk (TAR with FET). This study aims to analyze the renal protective effect of the ABO technique and to analyze the predictors of acute kidney injury (AKI) and continuous renal replacement therapy (CRRT) after TAR with FET.

Methods: Between August 2017 and September 2018, 247 patients who underwent TAR with FET were divided into $\mathrm{ABO}$ and moderate hypothermic circulatory arrest (MHCA) groups. The primary endpoint was postoperative AKI defined by the Kidney Disease Improving Global Outcomes (KDIGO) criteria. Multivariable logistic analysis was used to identify the predictors of AKI and CRRT after TAR with FET.

Results: With the application of the $\mathrm{ABO}$ technique, the circulatory arrest time was significantly shortened (ABO 4, IQR: 3-6 vs. MHCA 18, IQR: 16-20, P<0.001). Meanwhile, surgeons safely set the lowest nasopharyngeal temperature at a higher grade (ABO 28.1, IQR: 27.4-28.5 vs. MHCA 24.7, IQR: 24.1-25.1, $\mathrm{P}<0.001$ ). The peak serum creatinine ( $\mathrm{SCr}$ ) values within 48 hours after the surgery was lower in the $\mathrm{ABO}$ group than in the MHCA group (ABO 124, IQR: 97-173 vs. MHCA 146, IQR: 108-221, P=0.008). The distribution of AKI grade according to the KDIGO criteria differed between the two groups $(\mathrm{P}=0.04)$ : more patients in the ABO group were free from AKI (Grade 0) than patients in the MHCA group (33\% vs. $23.1 \%$ ), and the proportion of patients with high-grade AKI (Grades 2 and 3) in the ABO group was lower than that in the MHCA group (21\% vs. 32\%). The ABO technique was associated with reduced potential for AKI, but was not protective for CRRT.

Conclusions: The ABO technique significantly shortened the circulatory arrest time and safely elevated temperature, and provided better renal protection in patients undergoing TAR with FET. The ABO technique did not reverse the need for CRRT, nor did it reduce mortality or major adverse events.

Keywords: Aortic balloon occlusion technique (ABO technique); total arch replacement; frozen elephant trunk; acute kidney injury (AKI)

Submitted Dec 17, 2019. Accepted for publication Apr 06, 2020.

doi: 10.21037/acs-2019-0177

View this article at: http://dx.doi.org/10.21037/acs-2019-0177 


\section{Introduction}

Total aortic arch replacement with frozen elephant trunk (TAR with FET) has become a routine surgical procedure to treat complex aortic arch disease in China since it was first used in clinical practice for the treatment of type A aortic dissection (1). Moderate hypothermic circulatory arrest (MHCA) with selective cerebral perfusion (SCP) is widely used in aortic arch surgery and provides equivalent cerebral and visceral protection compared with deep hypothermic circulatory arrest (DHCA) with SCP (2-4). However, prolonged hypothermic circulatory arrest inevitably impacts distal viscera, especially the kidneys (5-7). Those with postoperative renal dysfunction have higher mortality and more complications than those without (8). We have reported that the aortic balloon occlusion $(\mathrm{ABO})$ technique shortens the circulatory arrest time to approximately 5 minutes compared to the previous average of 20-25 minutes and allows surgeons to safely perform operations at higher temperatures (9). However, there is no systemic data to indicate whether the $\mathrm{ABO}$ technique, which significantly shortens the MHCA time, improves the organ protective effect in TAR with FET. This study aimed to determine whether the $\mathrm{ABO}$ technique provides better distal visceral protection, especially for the kidney, than MHCA in patients who underwent TAR with FET, and to analyze the predictors of acute kidney injury (AKI) and continuous renal replacement therapy (CRRT) after TAR with FET.

\section{Methods}

\section{Patients}

A retrospective review of the Fuwai Hospital medical record system identified 247 patients who underwent TAR with FET in the vascular surgery center between August 2017 and September 2018. Patients were divided into two groups: the $\mathrm{ABO}$ and MHCA groups. This retrospective study was approved by the ethics committees of Fuwai Hospital, and written informed consent was waived.

\section{Operative technique}

The surgical procedures for the MHCA group have been described in previous articles (10). Patients were placed on cardiopulmonary bypass $(\mathrm{CPB})$ by cannulation of the right atrium and the right axillary artery. Cannulation of the right axillary artery was also used for antegrade SCP. During the cooling phase, aortic root procedures were performed, if necessary. Circulatory arrest was instituted when the nasopharyngeal temperature reached $24{ }^{\circ} \mathrm{C}$. Furthermore, antegrade SCP was started at a rate of approximately $5-8 \mathrm{~mL} /(\mathrm{kg} \cdot \mathrm{min})$. The aortic arch was transected between the left common carotid and left subclavian arteries. Next, the stented elephant trunk (Cronus, MicroPort Endovascular Shanghai Co, Ltd, China) was inserted into the true lumen of the descending aorta and released. At this time, surgeons needed approximately 20 minutes to anastomose the descending aorta and the stented elephant trunk to the 4-branched prosthetic graft (Vascutek Terumo, Tokyo, Japan; $28-30 \mathrm{~mm}$ in diameter). When anastomosis was complete, perfusion of the lower body was resumed through the perfusion limb of the 4-branched graft, and the CPB flow was gradually returned to one half of the full rate $\left(1.1-1.2 \mathrm{~L} / \mathrm{min} \cdot \mathrm{m}^{-2}\right)$. Epiaortic vessel reconstruction, proximal arch anastomosis, rewarming and closure completed the procedure.

The ABO technique has been described previously (9). It is a practical improvement based on conventional surgical procedures and fully utilizes the advantages of the frozen elephant trunk, namely, the metal "skeleton" and can be performed on any patient treated with an FET. The main difference from MHCA lies in the CPB management methods used to complete distal aortic arch anastomosis. Patients in the $\mathrm{ABO}$ group were placed on CPB by cannulation of the femoral artery in addition to the right axillary artery and right atrium. Cannulation of the right axillary artery was also used for antegrade SCP. We prepared the ABO device as shown in Figure $1 A$. When the patients temperature reaches to $28^{\circ} \mathrm{C}$, 3-5 min of MHCA is used to release of the stent graft into the true lumen of the descending aorta (Figure 1B). Once the stent is released, the aortic balloon (Coda Balloon Catheter, Cook Incorporated, Bloomington, IN, USA) with the sheath (W.L. Gore \& Associates, Inc., Flagstaff, AZ, USA) is deployed into the metal part of the stented graft and $40-45 \mathrm{~mL}$ of saline was injected into the balloon (Figure 1C). Perfusion of the lower body is then resumed through the femoral artery and the CPB flow is gradually returned to one half of the full rate $\left(1.1-1.2 \mathrm{~L} / \mathrm{min} \cdot \mathrm{m}^{-2}\right)$. The surgeon is then able to reconstruct the distal aortic arch with perfusion of the lower body (Figure 1D). The reaminder of the operation is the same as for patients treated with MHCA alone. Since cannulation of the femoral artery is part of our $\mathrm{ABO}$ technique, we listed severe malperfusion of the bilateral lower limb as a contraindication. Furthermore, 

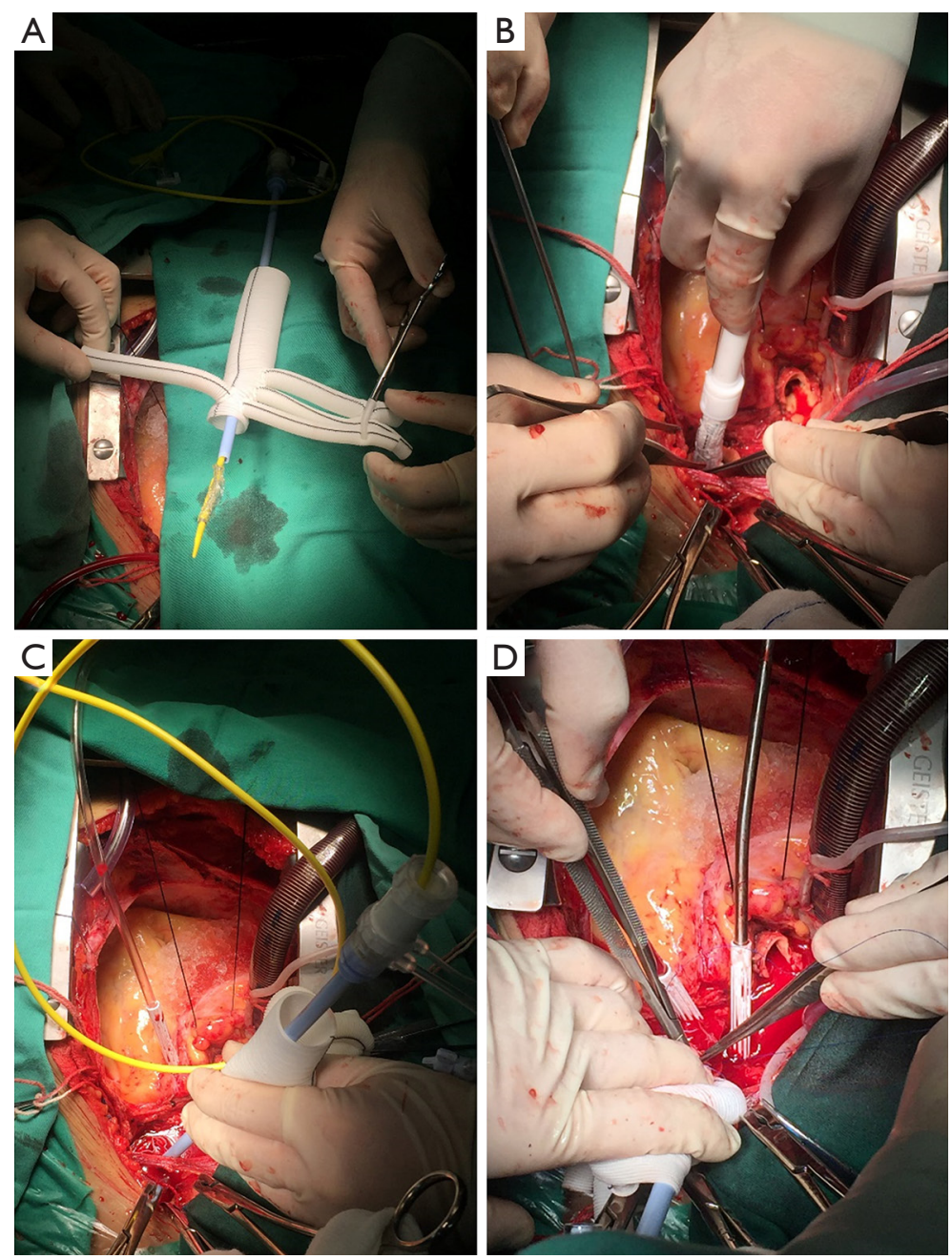

Figure 1 Main procedures of the aortic balloon occlusion technique. (A) The aortic balloon in a sheath was passed through the trimmed 4-branched graft in advance; (B) during selective cerebral perfusion, the stented elephant trunk was inserted into the true lumen of the descending aorta; (C) the aortic balloon with the sheath was deployed into the metal part of the stented graft and inflated with saline. Next, perfusion of the lower body was resumed from the femoral artery; (D) the 4-branched prosthetic graft was anastomosed with the descending aorta and stented elephant trunk with perfusion of the brain and lower body simultaneously.

habits of the surgeons played an important role in whether the $\mathrm{ABO}$ technique was used in the operation.

\section{Endpoints and data definition}

The primary endpoint was postoperative AKI defined by the Kidney Disease Improving Global Outcomes (KDIGO) criteria with slight modifications (Table 1). Postoperative AKI was defined as a postoperative serum creatinine (SCr) increase of more than $50 \%$ of baseline or if there was an increase of $26.5 \mu \mathrm{mol} / \mathrm{L}(0.3 \mathrm{mg} / \mathrm{dL})$ at 48 hours postoperatively. Preoperative SCr values nearest the time of surgery were used as the baseline SCr levels. The highest SCr values within 48 hours after surgery were collected. Urine output was excluded because of the difficulty and inaccuracy of the retrospective data.

The secondary endpoints included intraoperative transfusion, duration of mechanical ventilation, ICU and hospital lengths of stay, and adverse clinical outcomes, such as in-hospital mortality, reoperation for hemostasis, stroke, paraplegia, the use of intra-aortic balloon pump (IABP) and tracheotomy.

Demographic data, information on concomitant procedures and parameters concerning $\mathrm{CPB}$ were also 


\begin{tabular}{|c|c|}
\hline 0 & $<1.5$ times baseline and $0.3 \mathrm{mg} / \mathrm{dL}(26.5 \mu \mathrm{mol} / \mathrm{L})$ increase \\
\hline 1 & $1.5-1.9$ times baseline or $\geq 0.3 \mathrm{mg} / \mathrm{dL}(26.5 \mu \mathrm{mol} / \mathrm{L})$ increase \\
\hline 2 & 2.0-2.9 times baseline \\
\hline
\end{tabular}

collected.

Malperfusion syndrome can involve all organs of the body. Myocardial malperfusion was defined as acute myocardial infarction with ST segment elevation and a significantly increased value of serum cardiac troponin I. Cerebral and gastrointestinal malperfusion were defined as alteration in mental status and symptoms of abdominal pain or distention, respectively. Limb malperfusion was defined as the absence of pulse with limb compromise.

\section{Statistical analysis}

Descriptive statistics for categorical variables are reported as the frequency and percentage, whereas continuous variables are reported as the mean \pm standard deviation (SD) or median with interquartile range (IQR), depending on a normal distribution. Disordinal categorical variables were compared with the Pearson $\chi^{2}$ test or Fisher's exact test, and ordinal categorical variables were compared with the MannWhitney U test. Continuous variables were compared with Student's $t$ test or Mann-Whitney $U$ test as appropriate. Logistic regression models were used to find the protective and risk factors for AKI and CRRT after TAR with FET. All potential covariates of interest were included in a univariable logistic regression model. The multivariable logistic regression model included significant variables $(\mathrm{P}<0.1)$ in univariable logistic regression. For all analyses (beyond the univariate analysis), a $\mathrm{P}$ value of $<0.05$ was considered to be statistically significant, and all statistical tests were 2-sided. Data were analyzed using SPSS, version 21.0 (SPSS, Inc., Chicago, IL, USA).

\section{Results}

\section{Demographic data}

The mean age of all 247 patients was $47.8 \pm 10.9$ years (range 18 to 77$)$, and $76.1 \%$ were male $(\mathrm{n}=188)$. The $\mathrm{ABO}$ technique was performed in $100(40.5 \%)$ patients, and the other 147 (59.5\%) patients underwent surgery with MHCA. As described in Table 2, few significant differences in the baseline data existed between the groups, except for age (ABO 49.8 \pm 12.5 years vs. MHCA 46.4 \pm 9.6 years, $\mathrm{P}=0.02$ ).

\section{Operative characteristics}

Table 3 demonstrates the concomitant procedure data and $\mathrm{CPB}$ information. There was an equivalent percentage of concomitant procedures between the two groups. The patients in the $\mathrm{ABO}$ group underwent circulatory arrest at warmer nasopharyngeal (ABO 28.1, IQR: 27.4-28.5 vs. MHCA 24.7, IQR: 24.1-25.1, P<0.001) and bladder (ABO 29.2, IQR: 28.5-29.9 vs. MHCA 27.2, IQR: 26.3-28.2, $\mathrm{P}<0.001)$ temperatures than those in the MHCA group. Additionally, circulatory arrest time (ABO 4, IQR: 3-6 vs. MHCA 18, IQR: $16-20, \mathrm{P}<0.001)$ of the ABO group was significantly shorter than that of the MHCA group. There was no significant difference in the CPB time (ABO 170, IQR: 148-200 vs. MHCA 161, IQR: 142-193, $\mathrm{P}=0.13$ ) or aortic cross clamp time (ABO 111, IQR: 93-134 vs. MHCA 108, IQR: 89-128, $\mathrm{P}=0.08$ ) between the two groups.

\section{Primary and secondary endpoints}

Table 4 shows the main outcomes of this study. The preoperative SCr values were similar between the two groups (ABO 82, IQR: 69-104 vs. MHCA 85, IQR: 68-112, $\mathrm{P}=0.31)$. In the $\mathrm{ABO}$ group, peak postoperative $\mathrm{SCr}$ values (ABO 124, IQR: 97-173 vs. MHCA 146, IQR: 108-221, $\mathrm{P}=0.008)$ and maximum perioperative $\mathrm{SCr}$ variation $(\mathrm{ABO}$ $61.5 \pm 78.7$ vs. MHCA $83.7 \pm 89.7, \mathrm{P}=0.004)$ were significantly lower than those in the MHCA group. According to the KDIGO criteria, there was a significant difference in postoperative AKI between the two groups $(\mathrm{P}=0.04)$ : more 
Table 2 demographics and comorbidities

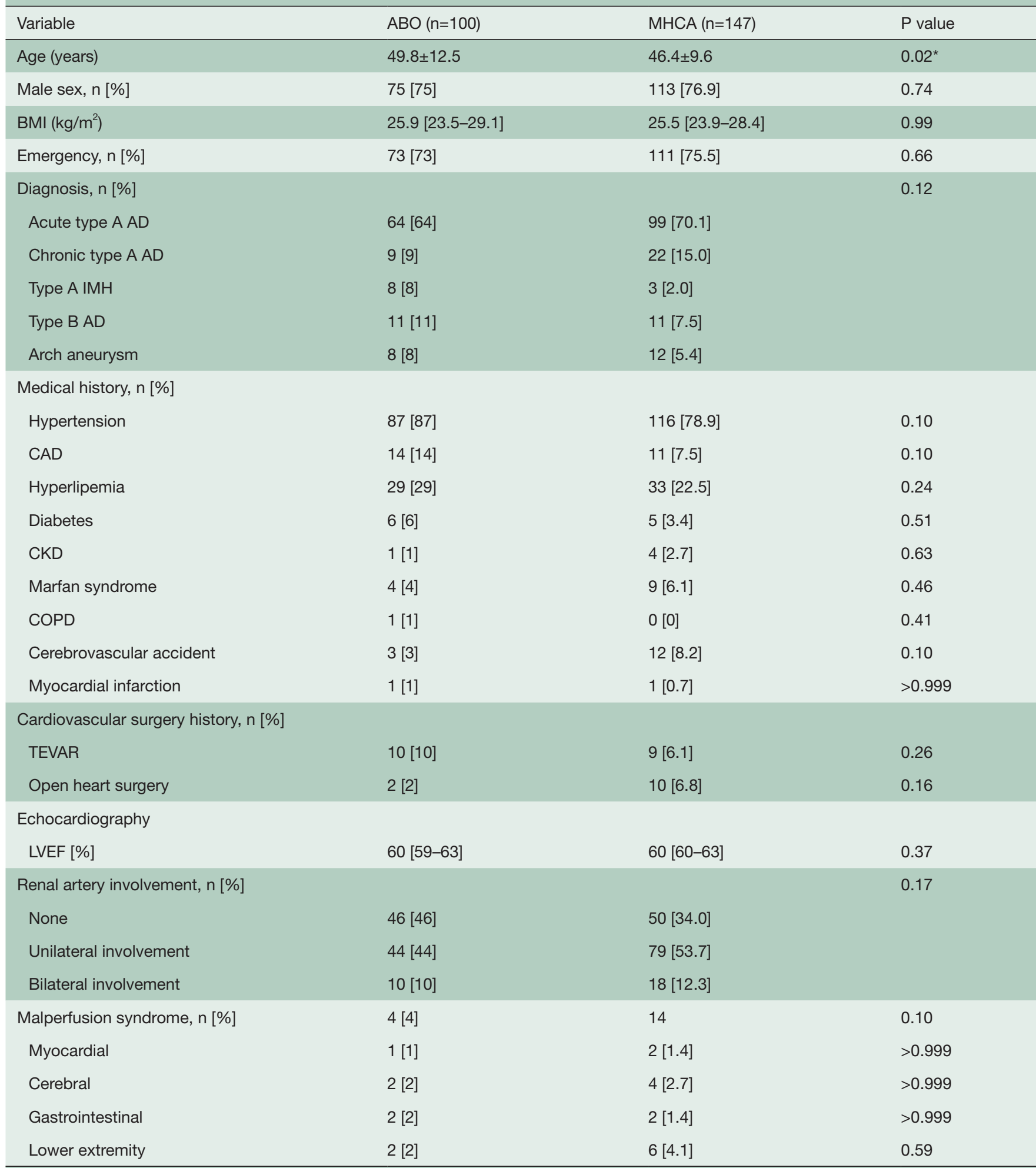

*, $\mathrm{P}<0.05$. $\mathrm{ABO}$, aortic balloon occlusion technique; $\mathrm{AD}$, aortic dissection; $\mathrm{BMI}$, body mass index; CAD, coronary artery disease; CKD, chronic kidney disease; COPD, chronic obstructive pulmonary disease; IMH, intramural hematoma; LVEF, left ventricular ejection fraction; MHCA, moderate hypothermic circulatory arrest; TEVAR, thoracic endovascular aortic repair. 


\begin{tabular}{|c|c|c|c|}
\hline Variables & ABO $(n=100)$ & MHCA $(n=147)$ & $P$ value \\
\hline \multicolumn{4}{|l|}{ Concomitant procedures, n [\%] } \\
\hline Commissural resuspension & $26[26]$ & 35 [23.8] & 0.70 \\
\hline Bentall procedure & 20 [20] & 33 [22.5] & 0.65 \\
\hline Wheat procedure & 7 [7] & $4[2.7]$ & 0.20 \\
\hline David procedure & $0[0]$ & 3 [2.0] & 0.40 \\
\hline CABG & 13 [13] & 24 [16.3] & 0.47 \\
\hline Ascending aorta-femoral artery bypass & $4[4]$ & $13[8.8]$ & 0.14 \\
\hline Others & 2 [2] & $4[2.7]$ & 0.98 \\
\hline CPB time (min) & $170[148-200]$ & $161[142-193]$ & 0.13 \\
\hline Aortic cross clamp time (min) & $111[93-134]$ & $108[89-128]$ & 0.08 \\
\hline Circulatory arrest time (min) & 4 [3-6] & 18 [16-20] & $<0.001^{\star \star \star}$ \\
\hline Lowest nasopharyngeal temperature $\left({ }^{\circ} \mathrm{C}\right)$ & $28.1[27.4-28.5]$ & $24.7[24.1-25.1]$ & $<0.001^{\star \star \star}$ \\
\hline Lowest bladder temperature $\left({ }^{\circ} \mathrm{C}\right)$ & $29.2[28.5-29.9]$ & 27.2 [26.3-28.2] & $<0.001^{\star \star \star}$ \\
\hline
\end{tabular}

patients in the $\mathrm{ABO}$ group (ABO 33\% vs. MHCA 23.1\%) avoided AKI and more patients in the MHCA group (ABO 21\% vs. MHCA 32\%) were diagnosed with highgrade (Grade 2 and Grade 3) AKI. The number of patients diagnosed with Grade $1 \mathrm{AKI}$ was similar between the two groups (ABO 46\% vs. MHCA 44.9\%). Approximately 8\% of patients needed CRRT postoperatively, and there was no significant difference between the two groups (ABO $8 \%$ vs. MHCA $8.2 \%, \mathrm{P}=0.96$ ). Both the rate (ABO $8 \%$ vs. MHCA $26.5 \%, \mathrm{P}<0.001$ ) and amount (ABO 0, IQR: 0-0 vs. MHCA 0, IQR: $0-1, \mathrm{P}<0.001$ ) of packed red blood cells (PRBCs) used in the $\mathrm{ABO}$ group were lower than those used in the MHCA group. There was no significant difference in ICU or hospital length of stay, or in the incidence of major adverse events between the two groups. Although there was no significant difference, the proportion of ventilation time less than 12 hours in the $\mathrm{ABO}$ group was higher than the proportion in the MHCA group (ABO 18\% vs. MHCA $11.6 \%, \mathrm{P}=0.15)$.

\section{Regression analysis}

The regression analysis results are provided in Table 5. Several variables were associated with AKI and CRRT according to univariable analysis $(\mathrm{P}<0.1)$. For $\mathrm{AKI}$, we found that the $\mathrm{ABO}$ technique (OR 0.52; 95\% CI: 0.28-0.96; $\mathrm{P}=0.03)$ and high preoperative SCr values $(>200 \mu \mathrm{mol} / \mathrm{L})$ (OR 0.18; 95\% CI: 0.04-0.83; $\mathrm{P}=0.03$ ) were associated with lower odds of postoperative AKI, while older age (OR 1.04; 95\% CI: 1.01-1.07; $\mathrm{P}=0.003)$, elevated body mass index (BMI) (OR 1.12; 95\% CI: 1.03-1.22; $\mathrm{P}=0.006)$ and renal artery involvement (OR 1.83; 95\% CI: 1.01-3.34; $\mathrm{P}=0.04$ ) were identified as independent risk factors for postoperative AKI according to multivariable analysis. For CRRT, we found that female sex (OR 4.00; 95\% CI: 1.09-14.70; $\mathrm{P}=0.04$ ), diabetes (OR 7.18; 95\% CI: 1.16-44.35; $\mathrm{P}=0.03$ ), malperfusion of gastrointestinal tract (OR 115.67; 95\% CI: 6.66-2,009.68; $\mathrm{P}=0.001$ ) or lower extremity (OR 18.99; 95\% CI: 2.66-135.35; $\mathrm{P}=0.003$ ), CPB time (OR 1.02; 95\% CI: $1.01-1.03 ; \mathrm{P}=0.001)$ and concomitant CABG (OR 6.54; 95\% CI: 1.86-23.00; $\mathrm{P}=0.003)$ were independent risk factors for CRRT according to multivariable analysis.

\section{Discussion}

In our study, we found that the $\mathrm{ABO}$ technique was an effective protective strategy for the kidney during the TAR with FET procedure and was also a protective factor for AKI. However, it was not a protective factor for CRRT after TAR with FET. The ABO technique also reduced 


\begin{tabular}{|c|c|c|c|}
\hline Variables & $A B O(n=100)$ & MHCA $(n=147)$ & $P$ value \\
\hline \multicolumn{4}{|l|}{ Kidney } \\
\hline Preoperative SCr ( $\mu \mathrm{mol} / \mathrm{L})$ & 82 [69-104] & 85 [68-112] & 0.31 \\
\hline Peak postoperative SCr in 48 hours ( $\mu \mathrm{mol} / \mathrm{L}$ ) & 124 [97-173] & $146[108-221]$ & $0.008^{* \star}$ \\
\hline Max perioperative SCr variation ( $\mu \mathrm{mol} / \mathrm{L})$ & $61.5 \pm 78.7$ & $83.7 \pm 89.7$ & $0.004^{* *}$ \\
\hline Grade of AKI, $n$ [\%] & & & 0.048 \\
\hline Grade 0 & 33 [33] & $34[23.1]$ & \\
\hline Grade I & $46[46]$ & 66 [44.9] & \\
\hline Grade II & $10[10]$ & 28 [19.1] & \\
\hline Grade III & $11[11]$ & 19 [12.9] & \\
\hline CRRT, n [\%] & $8[8]$ & $12[8.2]$ & 0.96 \\
\hline \multicolumn{4}{|l|}{ Intraoperative transfusion } \\
\hline PRBC patients, $n$ [\%] & 8 [8] & 39 [26.5] & $<0.001^{\star \star *}$ \\
\hline PRBC amount (units) & $0[0-0]$ & $0[0-1]$ & $<0.001^{\star \star \star}$ \\
\hline FFP patients, $\mathrm{n}[\%]$ & $56[56]$ & $76[51.7]$ & 0.51 \\
\hline FFP amount $(\mathrm{mL})$ & $400[0-600]$ & $400[0-600]$ & 0.87 \\
\hline PLT patients, $\mathrm{n}[\%]$ & 69 [69] & $101[68.7]$ & 0.96 \\
\hline PLT amount (units) & $1[0-1]$ & $1[0-1]$ & 0.55 \\
\hline Ventilation time & 20 [12-39] & $24[15-52]$ & 0.27 \\
\hline Ventilation $<12$ hours, $\mathrm{n}$ [\%] & $18[18]$ & $17[11.6]$ & 0.15 \\
\hline ICU length of stay (d) & $4[3-6]$ & $4[2-6]$ & 0.56 \\
\hline Hospital length of stay (d) & $13[9-17]$ & $12[10-17]$ & 0.80 \\
\hline 30-day mortality, $n$ [\%] & $3[3]$ & $7[4.8]$ & 0.72 \\
\hline Reoperation for bleeding, $\mathrm{n}$ [\%] & $5[5]$ & $6[4.1]$ & 0.98 \\
\hline Stroke, $\mathrm{n}[\%]$ & $5[5]$ & $3[2.0]$ & 0.36 \\
\hline Paraplegia, n [\%] & $2[2]$ & $7[4.8]$ & 0.43 \\
\hline IABP, n [\%] & $1[1]$ & $3[2.0]$ & 0.90 \\
\hline Tracheotomy, n [\%] & $3[3]$ & 3 [2.0] & 0.95 \\
\hline
\end{tabular}

intraoperative PRBC use. Many predictors were identified to be independent risk factors for AKI and CRRT after TAR with FET.

In our cohort, the incidence of AKI in patients who underwent TAR with FET under MHCA with SCP was $76.9 \%$, similar to the incidence of $75.6 \%$ in a previous report from our hospital (2). The incidence of AKI after aortic arch operation varied from $13 \%$ to $45 \%$ in previously published literature, which was significantly lower than the incidence found in our report $(7,11,12)$. There are three possible reasons for this difference; a lack of consistent diagnostic criteria for AKI, different surgical procedures, 


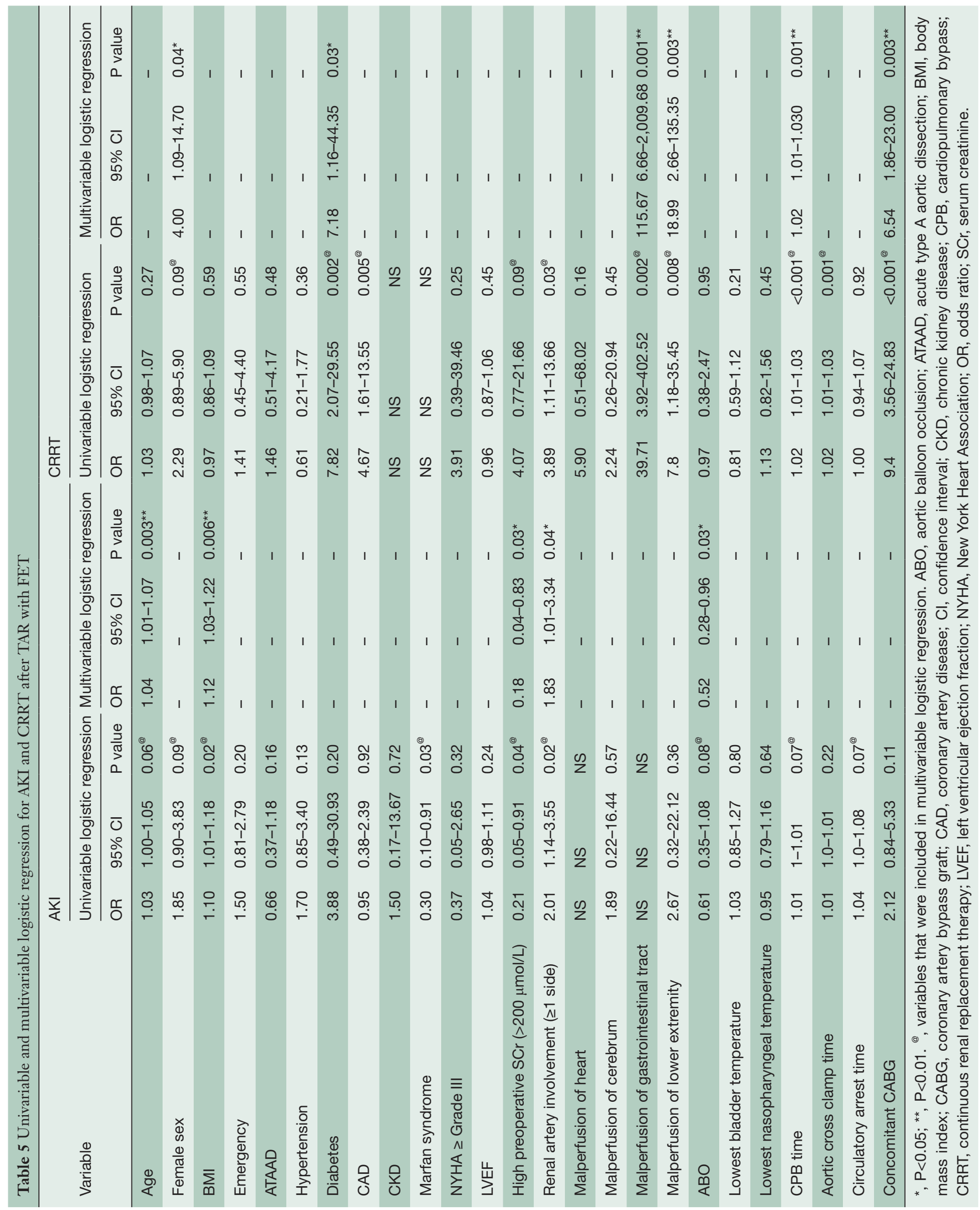


and an insufficient sample size. From our perspective, this was a reasonable study with a relatively large sample size and the use of specific KDIGO criteria to investigate AKI incidence after an identical procedure, TAR with FET.

With the development of surgical techniques and strategies for organ protection, TAR with FET under MHCA with SCP has achieved great outcomes with low mortality and fewer severe complications $(12,13)$. However, for further improvement of therapeutic efficacy, organ dysfunction caused by prolonged circulatory arrest remains a serious problem. According to recent reports, circulatory arrest time varies from 15 to $50 \mathrm{~min}$, and the temperature during circulatory arrest is mostly set at $22-26^{\circ} \mathrm{C}(2,13,14)$. The ABO technique reported by Xiaogang Sun was the first one to shorten the circulatory arrest time to approximately 5 minutes and safely raise the circulatory arrest temperature to approximately $28{ }^{\circ} \mathrm{C}(9)$. This was also the first study to systematically evaluate the renal protective effect of $\mathrm{ABO}$ technique.

By comparing perioperative SCr values and AKI incidence between the $\mathrm{ABO}$ and MHCA groups, we found that the $\mathrm{ABO}$ technique was associated with lower postoperative $\mathrm{SCr}$ values at 48 hours and a reduced incidence of postoperative AKI. Furthermore, multivariable logistic regression confirmed that the $\mathrm{ABO}$ technique was a protective factor for postoperative AKI. Therefore, we consider that $\mathrm{ABO}$ technique is an effective strategy to decrease the incidence and severity of AKI after surgery and to protect renal function despite a higher intraoperative core temperature. Tracing the history of organ protection in aortic surgery will provide a reasonable explanation. The application of DHCA represents the beginning of organ protection in aortic arch surgery (15). When SCP was used intraoperatively, it significantly reduced complications of the cerebrum and allowed surgeons to perform operations under moderate hypothermia $(2,4,11,16,17)$. Thus, just as SCP improves the protection of the cerebrum by maintaining cerebral perfusion, the main protective mechanism of the $\mathrm{ABO}$ technique is that this technique shortens the circulatory arrest time and improves renal perfusion.

Unfortunately, the $\mathrm{ABO}$ technique did not reduce the rate of CRRT. There may be two reasons for this outcome. Firstly, compared with the more than $16 \%$ incidence of CRRT after surgery reported in previous literature $(12,13)$, the rate of CRRT found in our cohort was extremely low. Thus, it is likely that the current study is underpowered to demonstrate a difference in CRRT requirement between the two groups given. Secondly, postoperative CRRT is associated with multiple factors. A previous study has reported that chronic kidney disease, lower limb ischemia, hyperkalemia, severe metabolic acidosis, heart failure be related to CRRT after surgical repair of type A aortic dissection (18). Our multivariable logistic regression found that many variables unrelated to the kidney were identified as independent risk factors for postoperative CRRT and our clinical practice also found that the indications for CRRT are varied, not just for severe AKI. Therefore, the ABO technique cannot change the outcome of these high-risk patients.

With the development of surgical technology and the increase in circulatory arrest temperature, transfusion in aortic surgery has already decreased, but is still higher than that of other cardiac surgeries $(2,11)$. In our study, we found that the rate and amount of intraoperative PRBC transfusion in the $\mathrm{ABO}$ group were further reduced, compared with those in the MHCA group. Some previous studies have reported that a longer hypothermic CPB time and DHCA are associated with a higher risk of postoperative bleeding volume and perioperative blood transfusion (19-21). The $\mathrm{ABO}$ technique raises the temperature during $\mathrm{CPB}$, which can reduce both the coagulopathy and perioperative bloodloss in the aortic arch surgery (19). However, the habits of surgeons are also an important factor influencing blood transfusion intraoperatively.

Our study found that the ventilation time in the ABO group was shorter than that of the MHCA group, and a higher percentage of patients in the $\mathrm{ABO}$ group were weaned from ventilation within 12 hours, although the difference was not significant. In our institute, protocols to wean patients from ventilation are normally initiated as soon as consciousness is regained. We interpret this outcome as a faster recovery of brain function from the impact of hypothermia and anesthesia. This phenomenon may be related to the elevated temperature and shortened time of the brain's dependence on SCP.

Many previous studies have reported risk and protective factors for AKI after aortic surgery (5-7,21). A higher baseline GFR (Glomerular Filtration Rate), and better LVEF were associated with lower odds of developing AKI, and a longer CPB time, male sex, increased age, elevated BMI and hypertension history were associated with increased odds of AKI. Our study also found that older age, elevated BMI and renal artery involvement were risk factors for AKI, which is similar to previous studies. In addition, the $\mathrm{ABO}$ technique is a protective factor for $\mathrm{AKI}$, 
convincingly proving that this technique can effectively protect renal function during the operation. Our study also found that higher preoperative SCr values $(>200 \mu \mathrm{mol} / \mathrm{L}$ ) was a protective factor for AKI, which was not only different from previous studies (6), but also seems to go against evidence from clinical practice. By further analyzing the clinical data of all patients, we found only 8 patients with preoperative SCr values greater than $200 \mu \mathrm{mol} / \mathrm{L}$. The proportion is so low that the result may be accidental. Among these 8 patients, 7 patients were diagnosed with aortic dissection, mainly with renal artery involvement (5 in 7 patients). TAR with FET is an effective strategy for opening up the true lumen of the aorta. After surgery, renal function improves with better perfusion, so postoperative AKI cannot be diagnosed owing to a decrease or mild increase in $\mathrm{SCr}$ values, which made higher preoperative $\mathrm{SCr}$ values a protective factor for $\mathrm{AKI}$ in the statistical analysis.

There are some limitations in our study. Owing to the retrospective design, there may be unaccounted bias in our results, but we have attempted to avoid this as much as possible. Four different cardiac surgeons performed all 247 surgeries. It is possible that inherent surgeon bias may have influenced the results. Much meaningful data cannot be collected retrospectively, which may affect the thorough evaluation of the $\mathrm{ABO}$ technique. The sample size was slightly insufficient. In the multivariate logistic regression for CRRT, the risk of overfitting should have been considered given that the number of CRRT events was low relative to many of the included variables. Consequently, further exploration, such as expanding the sample size and prospective research will be necessary.

\section{Conclusions}

The ABO technique significantly shortens circulatory arrest time and safely elevates temperature and provides better protection for the kidney in patients who undergo TAR with FET. However, this technique does not reverse the need for CRRT or reduce mortality and major adverse events. Therefore, the ABO technique should be applied for the unhurried suturing of distal anastomoses during the FET procedure. In addition, it may be better to exploit new frontiers to radically improve clinical outcomes.

\section{Acknowledgments}

Funding: This work was supported by Beijing Municipal Science and Technology Commission (Z181100001718197).

\section{Footnote}

Conflicts of Interest: The authors have no conflicts of interest to declare.

Ethical Statement: This retrospective study was approved by the ethics committees of Fuwai Hospital, and written informed consent was waived.

Open Access Statement: This is an Open Access article distributed in accordance with the Creative Commons Attribution-NonCommercial-NoDerivs 4.0 International License (CC BY-NC-ND 4.0), which permits the noncommercial replication and distribution of the article with the strict proviso that no changes or edits are made and the original work is properly cited (including links to both the formal publication through the relevant DOI and the license). See: https://creativecommons.org/licenses/by-nc-nd/4.0/.

\section{References}

1. Liu ZG, Sun LZ, Chang Q, et al. Should the "elephant trunk" be skeletonized? Total arch replacement combined with stented elephant trunk implantation for Stanford type A aortic dissection. J Thorac Cardiovasc Surg 2006;131:107-13.

2. Fang Z, Wang G, Liu Q, et al. Moderate and deep hypothermic circulatory arrest has a comparable effect on acute kidney injury after total arch replacement with frozen elephant trunk procedure in type A aortic dissection. Interact CardioVasc Thorac Surg 2019;29:130-6.

3. Gong M, Ma W, Guan X, et al. Moderate hypothermic circulatory arrest in total arch repair for acute type A aortic dissection: clinical safety and efficacy. J Thorac Dis 2016;8:925-33.

4. Keeling WB, Tian DH, Leshnower BG, et al. Safety of Moderate Hypothermia With Antegrade Cerebral Perfusion in Total Aortic Arch Replacement. Ann Thorac Surg 2018;105:54-61.

5. Zhou H, Wang G, Yang L, et al. Acute Kidney Injury After Total Arch Replacement Combined With Frozen Elephant Trunk Implantation: Incidence, Risk Factors, and Outcome. J Cardiothorac Vasc Anesth 2018;32:2210-7.

6. Roh GU, Lee JW, Nam SB, et al. Incidence and Risk Factors of Acute Kidney Injury After Thoracic Aortic Surgery for Acute Dissection. Ann Thorac Surg 2012;94:766-71. 
7. Nota H, Asai T, Suzuki T, et al. Risk factors for acute kidney injury in aortic arch surgery with selective cerebral perfusion and mild hypothermic lower body circulatory arrest. Interact CardioVasc Thorac Surg 2014;19:955-61.

8. Sasabuchi Y, Kimura N, Shiotsuka J, et al. Long-Term Survival in Patients With Acute Kidney Injury After Acute Type A Aortic Dissection Repair. Ann Thorac Surg 2016;102:2003-9.

9. Sun X, Guo H, Liu Y, et al. The aortic balloon occlusion technique in total arch replacement with frozen elephant trunk. Eur J Cardiothorac Surg 2019;55:1219-21.

10. Ma WG, Zhu JM, Zheng J, et al. Sun's procedure for complex aortic arch repair: total arch replacement using a tetrafurcate graft with stented elephant trunk implantation. Ann Cardiothorac Surg 2013;2:642-8.

11. Preventza O, Coselli JS, Garcia A, et al. Moderate hypothermia at warmer temperatures is safe in elective proximal and total arch surgery: Results in 665 patients. J Thorac Cardiovasc Surg 2017;153:1011-8.

12. Shrestha M, Haverich A, Martens A. Total aortic arch replacement with the frozen elephant trunk procedure in acute DeBakey type I aortic dissections. Eur J Cardiothorac Surg 2017;51:i29-i34.

13. Leone A, Beckmann E, Aandreas M, et al. Total aortic arch replacement with frozen elephant trunk technique: Results from two European institutes. J Thorac Cardiovasc Surg 2020;159:1201-11.

14. Shrestha M, Fleissner F, Ius F, et al. Total aortic arch replacement with frozen elephant trunk in acute type A aortic dissections: are we pushing the limits too far? Eur J

Cite this article as: Zhang B, Liu Y, Guo H, Li Y, Shi Y, Liang S, Liu H, Sun X. Renal protective effect of the aortic balloon occlusion technique in total arch replacement with frozen elephant trunk. Ann Cardiothorac Surg 2020;9(3):209219. doi: 10.21037/acs-2019-0177
Cardiothorac Surg 2015;47:361-6.

15. Griepp RB, Stinson EB, Hollingsworth JF, et al. Prosthetic replacement of the aortic arch. J Thorac Cardiovasc Surg 1975;70:1051-63.

16. Frist WH, Baldwin JC, Starnes VA, et al. A reconsideration of cerebral perfusion in aortic arch replacement. Ann Thorac Surg 1986;42:273-81.

17. Leshnower BG, Thourani VH, Halkos ME, et al. Moderate Versus Deep Hypothermia With Unilateral Selective Antegrade Cerebral Perfusion for Acute Type A Dissection. Ann Thorac Surg 2015;100:1563-8; discussion 1568-9.

18. Wu HB, Ma WG, Zhao HL, et al. Risk factors for continuous renal replacement therapy after surgical repair of type A aortic dissection. J Thorac Dis 2017;9:1126-32.

19. Keenan JE, Wang H, Gulack BC, et al. Does moderate hypothermia really carry less bleeding risk than deep hypothermia for circulatory arrest? A propensity-matched comparison in hemiarch replacement. J Thorac Cardiovasc Surg 2016;152:1559-1569.e2.

20. Mazzeffi M, Marotta M, Lin HM, et al. Duration of deep hypothermia during aortic surgery and the risk of perioperative blood transfusion. Ann Card Anaesth 2012;15:266-73.

21. Arnaoutakis GJ, Vallabhajosyula P, Bavaria JE, et al. The Impact of Deep Versus Moderate Hypothermia on Postoperative Kidney Function After Elective Aortic Hemiarch Repair. Ann Thorac Surg 2016;102:1313-21. 